\title{
Linx
}

Revue des linguistes de l'université Paris X Nanterre

$8 \mid 1996$

Du dire et du discours

\section{Défaut du dire, dire du défaut : les mots du silence}

Jacqueline Authier-Revuz

\section{OpenEdition}

Journals

Édition électronique

URL : http://journals.openedition.org/linx/1137

DOI : 10.4000/linx.1137

ISSN : 2118-9692

\section{Éditeur}

Presses universitaires de Paris Nanterre

\section{Édition imprimée}

Date de publication : 1 septembre 1996

Pagination : 25-40

ISSN : 0246-8743

\section{Référence électronique}

Jacqueline Authier-Revuz, " Défaut du dire, dire du défaut : les mots du silence », Linx [En ligne],

8| 1996, mis en ligne le 24 juillet 2012, consulté le 19 avril 2019. URL : http://journals.openedition.org/ linx/1137 ; DOI : 10.4000/linx.1137

Ce document a été généré automatiquement le 19 avril 2019.

Département de Sciences du langage, Université Paris Ouest 


\title{
Défaut du dire, dire du défaut : les mots du silence ${ }^{1}$
}

\author{
Jacqueline Authier-Revuz
}

1 De la non-coïncidence foncière entre les deux ordres hétérogènes que la nomination superpose - celui, relevant du général, du fini, du discret des signes, et celui relevant du singulier, de l'infini, du continu des "choses" -, de ce qu'on a appelé le "défaut de prise de la lettre sur l'objet"2, surgit, au principe même de la nomination, la dimension d'une perte, d'un "manque à nommer". Et c'est de ce manque à nommer - qui, pour le sujet parlant est singulièrement manque à se nommer, manque à dire la vérité qui "ne se dit pas toute parce que les mots y manquent" (Lacan) - que structurellement se constitue le sujet, dans un irréductible écart à lui-même, sujet de ce qu'il est parlant et par conséquent de ce qu'il est manquant.

2 Combien est insistante, pour l'être parlant, la blessure que lui inflige le "ratage" inhérent à la nomination, c'est ce dont témoigne la constance des fictions consolatrices qui y répondent - inépuisable inventaire des langues imaginaires les plus hétéroclites, mais qui, dans l'avant mythique d'un âge d'or ou d'un paradis perdu de l'humanité ou d'une enfance, dans l'ailleurs rêvé de contrées lointaines, inconnues ou utopiques, dans l'après projeté par les constructeurs de langues artificielles, se rejoignent en ce qu'elles nomment "bien" les choses, sans perte, sans écart, restituant par là-même aux sujets, fantasmatiquement, cette coïncidence à eux-mêmes que, constitutivement, leur interdit leur langue réelle.

3 Autre tentative - tentation - d'échapper au langage réel et à la perte qui est en lui, celle, diversement incarnée, de la non-parole : à ce qu'il y a, à la fois inévitable et inacceptable, de non-dit en tout dire, répondre par un radical non-dire; à cette part de silence qui traverse, la creusant, toute nomination, opposer le plein (le vide) de la non-nomination. C'est, ainsi, l'appel à la musique de Nietsche, "Chante ! Ne parle plus !", dans un renoncement à lutter avec la "maladie du langage" ${ }^{\text {; }}$ c'est, anéantissement et complétude, le silence de l'expérience mystique; c'est encore, évoquée par Borges dans ses Fictions, la hautaine et vertigineuse figure de Funes, muré dans son exigence impossible d'une "langue" infinie - une non-langue donc - à même de donner à chaque 
singularité son nom "propre" : "Non seulement, il lui était difficile de comprendre que le symbole générique chien embrassât tant d'individus dissemblables et de formes diverses ; cela le gênait que le chien de trois heures quatorze (vu de profil) eût le même nom que le chien de trois heures un quart (vu de face)."4

Si les langues imaginaires ou le silence répondent par la mise en place, fictive, d'un lieu autre, à la blessure du langage, c'est comme réponse inverse que peut être saisie la littérature, pratique qui n'est que de langage, inscrite toute entière, au lieu même de l'écart, dans ces mots qui sont manquants. A l'analyse de Barthes dans sa Leçon: "De ce qu'il n'y a point parallélisme entre le réel et le langage lui-même les hommes ne prennent pas leur parti, et c'est ce refus, peut-être aussi vieux que le langage lui-même, qui produit, dans un affairement incessant, la littérature" ${ }^{5}$,répond celle de D. Oster qui, évoquant "l'effrayante disparité des mots et des choses [et] cette distance, cet intervalle,

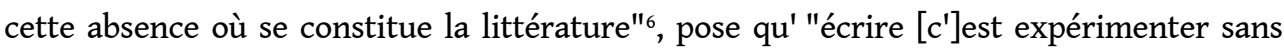
satiété ni contentement l'intervalle", ou encore, comme l'aveu d'un dénuement, cette phrase, dans Voleurs de mots, de M. Schneider: "On écrit surtout parce que les mots manquent."7 qui réveille le souvenir de cette plainte de Flaubert, adressée à un de ses correspondants : "A chaque ligne, à chaque mot, la langue me manque"8.

En deçà de la radicalité de ces "réponses" à la blessure du défaut du dire - le rêve du dire sans défaut, le silence du non-dire, l'écriture comme adhésion à la blessure du dire s'ouvre le champ de la négociation quotidienne des énonciateurs dans leur dire - leurs paroles, leurs écrits les plus divers - avec ce défaut qui l'affecte, champ où se déploie, à travers de multiples figures, une autre réponse consistant à accompagner le dire du dire de son défaut.

6 Cette réponse, c'est au fil du dire, ponctuellement, qu'on la saisit, dans les "boucles" d'un dire revenant réflexivement sur un point de son déroulement ${ }^{9}$, pour, le temps de cette boucle - celui d'une interrogation: est-ce le mot?, d'une critique : le mot ne convient pas, d'une acceptation: ce que j'appellerai faute de mieux, d'un renoncement: je ne trouve pas le mot, etc. suspendre le "qui va de soi" sur le mode duquel semble se donner ordinairement la nomination, en y inscrivant explicitement $d u$ défaut.

7 La rencontre par l'énonciateur, dans son dire, de l'écart entre les mots et les choses n'est pas la seule dont témoignent les "boucles" du dire. A travers la variété des reflets dont les énonciateurs accompagnent localement leur dire, ce sont toutes les difficultés, toutes les failles inhérentes au fait de dire qui apparaissent. Ainsi, la dépossession inscrite dans "nos" mots par le fait que ce sont ceux "des autres" que nous ne faisons qu'emprunter, chargés de sens par cet ailleurs - dans les X, comme on dit, par exemple -, par le fait aussi que leur sens nous échappe, dans leur inarrêtable équivocité - dans les $X$ au sens $p, X$ à tous les sens $d u$ mot, ... -, ou encore nous échappe en cet autre, irréductiblement différent, l'interlocuteur, auquel nous les adressons - dans les X si vous voulez, si vous voyez ce que je veux dire, ... - .

Soulignant d'un même mouvement la faille éprouvée dans le dire et le geste de sa suture, de sa "reprise" par l'énonciateur, toute forme de dédoublement local du dire par un autocommentaire apparait, contradictoirement, comme une "couture apparente" sur le tissu $\mathrm{du}$ dire ; mais pour les formes qui nous retiennent $\mathrm{ici}^{10}$, inscrites dans l'écart entre les mots et les choses, le paradoxe est que, spécifiquement, c'est à un manque de mots que répond ce surplus de mots que la boucle vient greffer en un point du fil du dire pour y nommer le manque, ouvrant le dire, par du dit, sur ce qu'il ne dit pas, faisant résonner, dans d'autres mots en plus, cette part de silence qui s'éprouve aux mots. 
9 Le parcours qui suit tente de faire apparaître, saisie à travers les formes mêmes, "au ras des mots", la variété de ces figures d'auto-commentaire qui, disant le défaut du dire, le disent sous des angles, avec des accents, sur des modes divers. Au-delà, ce qui apparaît, et que nous ne ferons qu'évoquer allusivement, c'est comment, dans un discours donné, pour un énonciateur donné, le choix fait dans cette variété - quelles figures sont élues préférentiellement, voire quelle figure exclusivement, jusque parfois au tic? - colore de façon singulière cette musique - ou ce bruit - du silence rencontré dans le dire, qui, sur des modes quantitativement très différents, ceux d'une musique occasionnelle, légère, ou insistante, lancinante, envahissante, accompagne le fil "principal" d'un discours.

Dans un premier type de représentation du défaut qui marque le dire, défaut est à prendre en un premier sens: celui du défaut, de l'imperfection, de l'anomalie que présente le dire, comme on dit qu'un vêtement, un bois, une personne $a$ un défaut, dont on peut décrire les caractéristiques. Cette description se fait sur deux versants (parfois associés en un seul commentaire) : ceux d'une caractérisation du mode de dire ou de l'élément $X$ lui-même.

11 Ainsi peut-on relever, face auX caractérisations inverses des $X$, je pèse mes mots ; $X$, je tiens au mot; $X, j$ 'assume le mot; etc., les images dépréciatives d'un mode de dire sommaire, nonachevé: dire placé dans les énoncés suivants (tirés des discours oraux et écrits les plus divers, des conversations familières aux textes théoriques ou littéraires les plus tenus) sous le signe de la rapidité, du "en gros", de la commodité, de l'aléa, du provisoire ou de la résignation, plus ou moins désinvolte, au défaut :

Elle est... réactionnaire, pour faire vite. (Conversation)

Voici donc le contact inopiné de l'histoire (on emploie ce mot par commodité) produisant sur la littérature deux effets finalement assez différents. (D. Oster, Passages de Zénon)

En quels sens, je le dis, à tout hasard, au pluriel, faut-il entendre cette relation d'appartenance à la même compagnie? (M. Arrivé, Jarry et Cie, 1985)

J'ai dit qu'ils ne se parlaient pas beaucoup; une sorte de contrainte étrange, inexplicable pesait sur eux aussitôt qu'ils se trouvaient seuls. (Je n'aime pas ce mot "inexplicable" et ne l'écris ici que par insuffisance provisoire). (A.Gide, Les FauxMonnayeurs)

12 Du côté des caractérisations du défaut que présente l'élément $\mathrm{X}, \mathrm{c}$ 'est une grande variété qui se présente : on y trouve de façon régulière de simples constats d'inadéquation du mot du type :

L'exposition (le mot ne convient sans doute pas) que vous proposez actuellement à

Beaubourg sur le thème des immatériaux. (J. Derrida, France Culture, 27-10-84)

ou bien des représentations qui spécifient la nature de l'écart entre le mot dit et la chose, et à travers lesquelles, de ce fait, la nomination $\mathrm{X}$, défectueuse, dès lors que son défaut est spécifié, n'est plus seulement le lieu de manque du mot juste, mais comme un point d'appui, à partir duquel s'amorce, dans l'espace ouvert entre le mot et la chose, par l'indication - très inégalement précise - d'une direction, d'une distance, d'une différence ..., un "balisage" du mot manquant. Comme des passerelles jetées sur l'écart ouvert dans la nomination entre le mot et la chose, les formes spécifiant cet écart peuvent partir, toujours avec la visée de "l'autre bord", de l'un des deux bords: formulant soit une prédication sur la chose - précisant la (les) propriété(s) du référent qui entre(nt) en conflit avec le mot $\mathrm{X}$-, sur le mode de $X$, si on peut dire $X$ pour quelque chose qui est..., comme dans : 
[...] nous entrons dans un café maure ; c'est de là que venait la musique. Des femmes arabes y dansent - si l'on peut appeler une danse ce monotone glissement. (A. Gide, L'immoraliste)

Dès qu'on aura trouvé une solution, enfin une solution entre guillemets, vu, qu'en fait c'est plutôt insoluble. (Conversation)

soit une prédication sur le mot - précisant de quelle façon il s'écarte de l'adéquation à la chose, à travers les figures dominantes des mots de sens trop étroit (qu'il faut "dilater" aux dimensions du réel à nommer) ou, au contraire, de sens trop général, imprécis (inapte à saisir strictement la chose), des mots trop forts ou au contraire trop faibles pour ce qu'ils nomment - cette dernière figure, celle de l'euphémisme explicité comme tel, apparaissant comme un mode rhétorique, stéréotypé, d'emphase sur l'intensité du réel nommé :

A ce moment ça redevient une question de sémantique en donnant au mot un sens un peu élastique. (Oral)

Ce n'est pas de l'adoption à proprement parler, euh de l'adoption au sens large, ils ont pris la charge, mais ce n'est pas légalisé, ça ne pouvait pas l'être d'ailleurs, je pense. (Oral)

Depuis Freud, de nombreux psychanalystes ont tenté de surmonter la difficulté qu'il y a pour les psychotiques (je n'aime pas ce terme trop généralisateur...) de développer un transfert analysable. (A. de Mijolla, in l'Événement du jeudi, 20.3.85)

Il n'a rien fait pendant le trimestre, enfin rien, c'est excessif, mais du coup ça patine un peu. (Oral)

Après le succès très relatif, et c'est un euphémisme, de la collection Rue Racine, lancée par Françoise Verny [...]. (Le Canard enchaîné, 22.6.88)

Avec une fréquence bien moindre, c'est un éventail varié de distorsions dans l'adéquation du mot à la chose qu'il nomme que l'on rencontre, figures de mots "déplacés" hors de leur lien "normal" au plan des choses, dans la métaphore, "l'analogie", l'antiphrase,

Des séparations institutionnalisées comme les crèches et les équivalents qui sont des éléments très importants qu'on pourrait dire antipsychotiques, enfin entre guillemets. C'est un peu métaphorique ce que je dis. Entre guillemets donc, vis-à-vis de l'enfant comme de la mère. (Conférence)

Ah, c'est un homme, pour parler par antiphrase, d'une ... exquise courtoisie... (Conversation)

ou bien de mots déplacés, au plan des mots, hors de leurs environnements "normaux", et associés en dénominations inusuelles, voire paradoxales ou contradictoires, c'est-à-dire problématiques quant à leur aptitude à saisir le plan des choses :

Si l'on peut dire qu'il n'y a pas de place pour le vague et l'à-peu-près dans la pratique mathématicienne, il faut aussi affirmer que la tâche principale de la mathématique est d'apprendre à parler (si l'on ose risquer cette alliance de mots), à parler avec rigueur des approximations. (G. Th. Guilbaud, Mathématiques et à-peuprès)

[...] quand on a l'oreille à peu près absolue, si on peut rapprocher ces deux expressions, [...]. (Conversation)

Le mouvement amorcé ici s'accuse dans les gloses où c'est la question même de l'adéquation qui est suspendue, dès lors qu'une des deux réalités, celle du sens ou celle de la chose, ferait (ou tendrait à faire) défaut, mettant en cause l'existence même du lien inhérent à la nomination. Ainsi à la mise en cause radicale de la coïncidence sémanticoréférentielle (du sens et de la chose), par "vidage" du sens,

J'aimais ses yeux, en effet, plus que tout peut-être. Ses yeux dilatés, bleus, brillants, durs, terribles - ces épithètes sont exécrables et ne disent rien - yeux immenses, bleus [...]. (P. Quignard, Le salon du Würtemberg)

Si on prend les analyses traditionnelles du phénomène, enfin, traditionnelle entre guillemets parce que le mot ne veut pas dire grand chose. (Conférence) 
[Althusser] possédait une extraordinaire capacité d'écouter la singularité de chacun et de la susciter. Le véritable Althusser, si cette expression a un sens, c'est d'abord cette capacité. (E. Balibar, Écrits pour Althusser)

répond celle qui opère ce vidage à partir de l'autre plan, celui du référent (de la chose) :

[...] il était bien obligé de parler d'abord des aspects codiques, c'est-à-dire collectifs transindividuels, qui assurent la communication au sens le plus simple du terme (à supposer que cela existe). (A. Culioli, Un linguiste devant la critique littéraire)

Le deuxième type de représentation du défaut du dire, s'inscrit du côté de l'autre valeur du mot défaut, complémentaire de la première, celui d'une absence, d'un manque, de ce qui fait défaut au dire - que ce soit "le" mot, le mot juste qui manque au dire, creusant la nomination de son absence, ou bien que ce soit l'énonciateur qui fasse, en quelque sorte, défaut à son dire du mot, comme "absenté" d'un dire qui se dérobe à sa pleine effectuation.

Sur le versant du mot qui manque, deux figures sont évoquées, celle de la défaillance de l'énonciateur, incapable de trouver "le" mot, ramenable à un je ne sais pas dire, et celle de la défaillance de la langue, ramenable à il n'y a pas de mot :

[...] il y a des gens qui sont très forts - et qui à mon avis sont peut-être peu nombreux - qui arrivent à troubler à distance par télépathie - télékinésie ou je ne sais pas comment vous dire - mais à troubler les personnes. (Emission télévisée sur la sorcellerie)

Deux phénomènes se sont produits. [...] D'une part, le régime que j'appellerai, faute de mieux, "soviétique", a implosé. (Entretien télévisé)

Ils ont fouillé dans mes affaires ! Ils ne m'ont laissé que mon pyjama ! [...] ils sont horribles, inhumains, [...] jamais je ne trouverai le mot exact ! Il n'existe pas ! (V. Valère, Le pavillon des enfants fous)

18 Sur l'autre versant, s'opposant au $X$, je dis bien $X$ qui redouble un dire $X$ de la représentation emphatique de son effectuation, répond, mettant en jeu toutes les ressources modales de la langue, un riche "nuancier" d'images du dire de X comme se faisant sur le mode de ne pas (ou pas tout à fait) se dire, dire s'effectuant sur le mode du presque, de l'à peine, sur le mode de n'être qu'une potentialité de dire au conditionnel, qu'une tentation de dire ou une hésitation à dire :

Érotique de la lecture ? Oui, à condition de ne jamais gommer la perversion et je dirai presque : la peur. (R. Barthes, Le grain de la voix)

Je me suis attelé à une analyse que j'ose à peine appeler linguistique tant elle était simpliste et maladroite [...]. (Entretiens de C. Lévi-Strauss et D. Eribon, De près et de loin)

[...ce livre] vient troubler une douce harmonie en rappelant, non sans un certain courage face à ce qu'on serait tenté d'appeler le totalitarisme mou de l'unanimisme, quelques vérités rugueuses. (M. Plon, in Frénésie, 8, 1989)

[...] nous réjouir pour ce qui semble être, oserai-je dire une réussite, et nous rappeler le chemin parcouru. (Allocution association locale)

et, enfin, ces deux modalités "irréalisantes" du dire : celle - modalité suspensive du dire d'un dire dont l'effectivité est représentée comme suspendue à une condition (en si) et celle - modalité annulatoire du dire - d'un dire s'effectuant sur le mode représenté de sa non-effectuation : $j^{\prime}$ allais dire $X$, je n'ose pas dire $X$

Il avait acquis une véritable virtuosité, si on peut employer ce mot dans ce cas, et ça a duré longtemps avant qu'il ne se fasse prendre. (Oral, à propos d'activités de pickpocket)

Si tu voyais comment il parle, si on peut appeler ça parler, à son assistante; c'est comme si c'était un robot. (Oral) 
[...] le mieux est peut-être de renvoyer à Bloomfield et à son compatriote et prédécesseur Peirce, lequel écrit, j'allais dire lumineusement: "Le problème de ce qu'est la 'signification' [...]". (R. Eluerd, La pragmatique linguistique)

Reconduit à Bâle par le fidèle Overbeck dès le 9 janvier 1889, le corps de Nietsche finira de mourir à Weimar le 25 août 1900 à midi. On ne sait pas grand chose, en fait, de cette large décennie de ce qu'on n'ose appeler encore, sa vie. (R.P. Droit, in Le Monde, 6.1.89)

19 Notons encore l'inflexion particulière attachée à ces formes - non rares - de dire du défaut du dire : celle qui, renvoyant le défaut d'une nomination $\mathrm{X}$ à un puisque/si il faut donner un nom à tout, ouvre dans celle-ci comme l'écart d'un regret, d'une nostalgie, d'une revendication du silence banni par la contrainte de dire - qui est loi de manque -, et comme une "fatigue" de nommer :

Sans doute que c'est le cas de parler de ...passion, puisqu'on ne peut pas laisser les choses sans mettre un nom dessus. (Oral, janvier 1986)

S'il est quelque chose, ce serait plutôt pervers, s'il faut vraiment mettre un nom dessus. (Oral, octobre 1986)

Une chose que le "féminisme" s'il faut donner un nom à tout, a apporté d'extraordinairement précieux aux femmes. (F. Nespo, Entretien avec S. Signoret, F. Magazine, août 1979)

20 Au-delà - ou en deçà - de cette diversité des figures de représentation du défaut qui marque le dire - spécifiant le défaut présent, la rapidité, l'imprécision, ... ou ce qui fait défaut, l'adhésion de l'énonciateur à son dire, le mot juste, ... - une forme retient l'attention : c'est, instituée par l'usage comme un des stéréotypes du dire du défaut du dire, la forme pour ainsi dire qui ne dit, pourtant, littéralement rien d'autre que ... le dire de X, sans aucun des éléments de caractérisation négative notés précédemment : le verbe dire y apparaît en effet sans expansion (adverbiale, modale, polarité négative, ...) et l'élément $\mathrm{X}$ y est représenté anaphoriquement par ainsi de la façon la plus neutre qui soit.

21 Accompagnant volontiers les inadéquations nettement repérables de métaphores ou de formulations de degré excessif, c'est très souvent à des écarts vagues, non aisément assignables, qu'elle renvoie, affectant le dire de $\mathrm{X}$ d'une non-coïncidence floue, comme d'un halo d'incertitude ou d'un sentiment diffus de coïncidence non-atteinte :

[...] dans les mythologies politiques, les identités collectives sont surinvesties, unifiées, substantialisées, pétrifiées enfin en objets de convictions, en assises de certitudes. Elles sont pour ainsi dire gelées et [...]. (P.A. Taguieff, in Mots, mars 1986) D.E. - Et qu'avez-vous fait?

G.D. - La même chose qu'avant. La retraite n'a pour ainsi dire rien changé dans ma vie. (G.Dumézil, Entretien avec D.Eribon, 1987)

[...] dans le cas de Dora, ce sont les rêves [...] qui constituent on l'a vu, les points d'ancrage - prenant pour ainsi dire la place qu'occupaient les symptômes à l'époque de Breuer. [...] Il arrive que le rêve ne doive pas être interprété comme la peinture de quelque chose, mais comme, pour ainsi dire, la représentation imagée des mots eux-mêmes. (O. Mannoni, Freud)

- Qu'est-ce qui vous fascinait dans ce cinéma-là?

- Son respect pour quelque chose de très, très simple, pour la vie quotidienne, pour ainsi dire. Et une façon de raconter la vie quotidienne, sans aucune sensation, sans histoire pour ainsi dire, parce que dans ces films-là, on ne peut même pas parler d'histoire. (Entretien avec W. Wenders, Télérama, 26-9-84)

22 Ainsi, est-ce une forme n'explicitant, au pied de la lettre, nul défaut, nul manque, nul écart, disant seulement le fait de dire, de nommer, qui apparaît comme forme stéréotypique du pur écart par inadéquation, c'est-à-dire du défaut du dire - comme si cette forme 
qui, disant, littéralement, le dire, a, en langue, la valeur de signifier l'écart du dire, témoignait d'un savoir, déposé dans la langue, de ce que le dire est écart.

Par sa spécificité littérale, cette forme qui, ne disant que le dire, dit le manque, se prête particulièrement, accompagnant répétitivement le fil d'un discours donné, à y dire - audelà du manque, ponctuel, d'un mot (ou du manque dans tel mot) - le mot, tout mot, comme manque. C'est le cas, à notre sens, pour les locuteurs qui, affectés de ce "tic de parole", y manifestent, de façon lancinante, plus que l'inadéquation particulière d'un certain nombre de nominations, la séparation qu'ils ne cessent d'éprouver, dans leur dire, d'avec les choses et d'avec eux-mêmes.

Apparaissent, dans leur différence, leur singularité, comme caractéristiques des discours qu'elles accompagnent de leur reflet, les images que ceux-ci produisent de leur manque à dire ; ces reflets du manque propres à chaque discours, quelques questions permettent d'en esquisser les contours : combien?, visant la place qu'occupe dans un discours le dire de son défaut, depuis les discours vierges de toute image du défaut de leurs mots, jusque, au contraire, aux discours incessamment occupés à répondre au manque qui s'ouvre en eux presque à chaque mot et, inévitablement, se rouvre à nouveau, récursivement, dans les mots mêmes de la "boucle" disant le manque ; où ?, localisant les lieux d'affleurement dans le discours de la non-coïncidence entre les mots et les choses, lorsque le sentiment de celle-ci impose au fil continu du discours l'accident de la greffe que constitue le commentaire, lieux qui dessinent dans le discours une "géographie de ses points sensibles", points de "nomination difficile" propres à un discours, où se trouvent soulignés ses tabous, ses impasses, ses points aveugles ou ses points d'exigence, déçue, de vérité de la nomination; comment?, spécifiant les types de formes par lesquelles, dans la variété que nous venons d'évoquer, le discours réfléchit, et "négocie", le défaut qu'il rencontre en lui-même.

Il n'est évidemment pas question, ici, d'illustrer cette spécificité des "dire du défaut du dire" à travers un large éventail de discours. C'est au champ littéraire que j'emprunterai l'exemple de trois écritures, au plein sens du terme, pour lesquelles, de façon frappante, le mouvement foncier qui porte toute écriture - répondre par des mots au manque qui est aux mots - s'accomplit, spécifiquement, dans la forme du "geste" de retour du dire sur luimême, l'écriture - constitutivement "dans" l'écart - avançant, doublée d'un dire de l'écart, dédoublée dans le reflet, ou l'écho, de son défaut: il en est ainsi, selon des modes singuliers, privilégiant des formes différentes de dire du manque à dire, de l'écriture de Pascal Quignard dans Le lecteur, de celle de Nathalie Sarraute dans, en particulier, L'usage de la parole, ou de Claude Simon dans La route des Flandres.

Accompagnant le fil du récit de P. Quignard - une enquête du narrateur qui, tâchant d'élucider le "mystère" de la "disparition" du Lecteur (déclinée en "absence, défaut, soustraction, retirement, ...") découvre dans cette "absence que porte le langage" le lieu où s'est produite cette absence au monde et à soi où a "disparu" le lecteur -, les formes qui disent le défaut du dire sont ici des formes brèves, figées, "discrètes" pourrait-on dire, comme si j'ose dire (contrairement aux gloses longues proliférant chez Nathalie Sarraute, dans l'espace largement ouvert entre mots et choses), des formes situant la faille sur le versant du rapport de l'énonciateur à son dire, du type si j'ose dire, je n'ose dire (et non sur celui de l'inadéquation du mot à la chose, du type le mot est mauvais, là encore largement occupé par les commentaires de Nathalie Sarraute), des formes enfin, relevant de ce que nous avons appelé modalités "irréalisantes" du dire, suspension en sij'ose dire, annulation en 
je n'ose dire (contrairement aux gloses confirmant, d'un je dis mal ou pour employer un mot approximatif, le fait même du dire), ainsi :

N'importe. Car quels que soient noms, expériences, espérances, ce qui demeure dans tous les cas je n'ose dire "acquis", c'est l'absence du langage : perte de toute foi ajoutée à la médiation du langage.

Mon fils rit aux éclats. [...] soudain il a cédé à un emportement que la facétie [...] ne justifiait plus, il s'est prêté à cet éclatement, à ces éclats. C'est cet éclatement qui l'a contraint à éclater en larmes. C'est dire qu'il a sangloté de ce qui ainsi n'est plus tout simplement de la joie. Je n'oserais dire : d'un sanglot qui excède la joie.

La dévoration par les livres, je me l'explique, si j'ose dire, comme l'espèce seconde d'une métempsycose entre l'absence d'une âme et l'absence d'un sens.

Ainsi, il découvrit le ciel , si j'ose dire, de son absence.

[...] la sorte de grâce que le langage [...] nous aurait fait une fois pour toutes qui est une assurance sans doute suspecte mais à laquelle donner cours est une promesse continuée, je pêcherais par excès en disant d'agrément, au moins d'angoisses relayées, de questions indéfiniment, c'est à dire au fil de la lecture, renouvelées et suspendues. (P.Quignard, Le lecteur)

Ainsi, apparait, à travers les formes signifiantes précises à travers lesquelles elle se réalise, et nettement différenciée de celui d'une écriture labourée, recouverte par sa lutte avec l'inadéquation (N.Sarraute), ou incessamment "posée" dans l'oscillation et l'incertitude de l'écart (C. Simon), un mode particulier - une tonalité propre d'affleurement au dire de l'écart qui le marque : mode discret mais incisif, aigu, de brèves altérations "en mineur" où le dire - comme "retiré", suspendu, annulé - se fait défaut à lui-même.

C'est sur un tout autre mode que l'écriture de Nathalie Sarraute apparait, elle-aussi, comme une écriture de l'écart: c'est, ici, dans d'incessants retours sur ses mots, une écriture taraudée par sa non-coïncidence qui se représente, "ouverte" - comme on dit un "chantier à ciel ouvert" - sur l'écart qu'elle éprouve, dénonce, mesure, parcourt, tente de réduire, retrouve ... entre les mots et les choses.

Dans Enfance, l'auteur inscrit dans l'impérieuse nécessité de saisir - c'est-à-dire nommer -, "tant bien que mal", "quelque chose d'encore informe" qui se dérobe, le pourquoi et le comment de son écriture; et le cheminement de son style, tenace, "trébuchant, tâtonnant, si honnête, si plein de repentir, qui approche l'objet avec des précautions pieuses" (Sartre), passe de façon cruciale par le dédoublement du dire, représentant - tout sauf léger, anodin, désinvolte, ... - le travail de nommer, inscrit, sérieux, grave, tendu, dans la non-coïncidence des mots aux choses, de soi-même à son dire. Et les formes qui, massivement, dominent dans ce constant mouvement réflexif sont des formes du retour en arrière dans la linéarité sur le dit, effectué, d'un élément, du type $X$, mais $X$ est inadéquat ; $X$, bien qu'il ne convienne pas (contrairement aux formes préposées du type si j'ose dire $X$, je n'ose dire $\mathrm{X}$ ), des formes ouvertes, $\mathrm{j}$ 'entends non figées, explorant explicitement cet écart singulier entre tel mot et la chose visée (et non pas ces formes figées, indiquant seulement qu'il y a "de l'écart", comme pour ainsi dire par exemple), des formes portant à la fois sur les mots - "gros, vagues, impuissants, indigents, brutaux, inadéquats, ..." - et sur le mode du dire - "être tout de même en droit de dire", "oser à peine murmurer", "osons dire quand même, il le faut bien" -, mettant en cause, dans leur défaillance, et la langue et l'énonciateur.

Citons quelques uns de ces retours sur soi, tirés de L'usage de la parole, et en particulier de l'ouverture de la nouvelle Esthétique, dont il faudrait citer des pages entières qui "n'avancent" qu'au prix de ces incessantes butées sur les mots : 
[...] laissant à travers lui passer comme une lumière diffuse, un rayonnement... pardonnez-moi ces mots indigents mais où pourrais-je en trouver d'autres? ... un doux, diffus rayonnement venu de fonds lointains [...].

Donc, la réponse arrive dans ce qu'elle a d'étrange ... Mais c'est un tort de dire : la réponse. Car les réponses qui présentent ce même caractère sont légion [...]. (L'usage de la parole)

Le lieu où cela s'est passé... mais comme "s'est passé" paraît peu convenir à ces moments, les plus effacés qui soient, les plus dénués d'importance, de conséquence [...] Renonçons donc à s'est passé... disons "a été vécu"... bien que cette expression puisse elle aussi sembler grandiloquente, outrée, tant ces moments paraissent peu mériter de faire partie de ce que nous nommons "notre vie". (Esthétique)

exemplaires de cette écriture adéquate à son objet, c'est-à-dire ayant la forme de ce qu'elle dit, qui, parlant "de mots" - de leur poids, leur violence, leur gravité, leur défaillance, leur impuissance, saisis, "cristallisés", dans des récits "d'instants" de parole -, en parle en se retournant sans cesse sur ses propres mots, éprouvant en elle-même d'abord, incessamment - loin des quiétudes ou des jubilations de l'adéquation du mot à la chose et de l'adhésion de l'énonciateur à son dire - comme une souffrance de la nomination.

D'emblée, frappe aussi, dans La Route des Flandres, l'importance quantitative, dans l'écriture de Claude Simon, des formes représentant l'écart dans la nomination : présence insistante, répétitive voire obsédante, d'un commentaire participant intimement sur le mode d'une "pulsation", d'un accompagnement, à une écriture dont le mouvement, toujours relancé par l'instabilité, l'incertitude, le non-un qui l'affecte, "donne forme" aux oscillations-vacillements de la mémoire et de l'identité.

Loin des "petits drames" de l'échec d'une nomination et des cheminements laborieux et divers inscrits dans l'espace entre mot et chose chez N. Sarraute, la représentation passe ici par des formes brèves, figées, répétitives - X ou plutôt $Y, X, c^{\prime} e s t-\grave{a}$-dire $Y, X$ pour ainsi dire - dont la continuelle reprise relève plutôt d'une "tonalité" de non-coïncidence intégrée au dire, constituant le mode, le régime, du dire.

Pour les premières, ou plutôt, c'est-à-dire, qui relèvent d'un ensemble de formes représentant la nomination comme "partagée" entre deux mots (non évoquées dans le parcours ci-dessus), on note que ce sont, dans cet ensemble, les formes les plus neutres, les plus discrètes, sans mise en scène du sujet énonciateur et des nuances modales détaillant ses hésitations (comme dans $X$, je devrais peut-être dire $Y$; X, irais-je jusqu'à dire $Y$ ?; ...), qui sont requises, prenant figure dans leur extrême répétitivité de phénomène consubstantiel au dire, partie intégrante de la machinerie qui le propulse, comme une respiration de la parole; comme, par exemple, dans

[...] en pleine retraite ou plutôt débâcle ou plutôt désastre au milieu de cette espèce de décomposition de tout [...].

[...] se défier l'un l'autre au péril de leur vie c'est-à-dire l'un prêt à (ou plutôt brûlant, dévoré par l'envie ou plutôt le besoin ou plutôt par la nécessité de) commettre un crime [...].

[...] alors j'éloignai le miroir, mon ou plutôt ce visage de méduse basculant s'envolant comme aspiré par le fond ombreux marron de la grange, [...] et à la place je les vis à l'autre bout de l'écurie, palabrant ou plutôt se taisant c'est-à-dire échangeant du silence comme d'autres échangent des paroles c'est-à-dire une certaine espèce de silence qu'ils étaient les seuls à comprendre [...].

Du côté de la représentation du défaut d'un terme, le pour ainsi dire (avec pas moins de 60 occurrences dans le roman) est la forme tout à fait dominante: contrairement aux modalités de "semi-dire", irréalisé, notées chez P. Quignard, c'est un dire qui s'effectue qui 
est représenté, et contrairement aux explicitations de la nature de l'inadéquation propres à N. Sarraute, c'est, associé à une profusion de modalisateurs d'approximation comme en quelque sorte, une sorte de, une espèce de, et de saisies par analogies en comme, comme si, à la façon de, le fait, brut, qu'il y ait de l'écart qui est représenté ici, et non pas tel ou tel type d'écart.

Ainsi, par exemple :

[...] il lui semblait toujours la voir, là où elle s'était tenue l'instant d'avant, ou plutôt la sentir, la percevoir comme une sorte d'empreinte persistante, irréelle, laissée moins sur sa rétine (il l'avait si peu, si mal vue) que, pour ainsi dire, en luimême : une chose tiède, blanche comme le lait qu'elle venait de tirer [...] il devait être tard et pourtant le jour était encore de ce même blanc sale dans lequel elle avait disparu, qui l'avait absorbée et pour ainsi dire épongée dans l'aube chargée d'eau ou plutôt imbibée imprégnée comme une étoffe comme [...].

[...] c'était comme un crépitement alternant se rattrapant se superposant se confondant par moment [...] et cela ainsi de suite, la guerre pour ainsi dire étale pour ainsi dire paisible autour de nous [...].

[...] et ce dut être par là que je le vis pour la première fois [...] le fixant à travers cette sorte de demi-sommeil, cette sorte de vase marron dans laquelle j'étais pour ainsi dire englué, et peut-être parce que nous dûmes faire un détour pour l'éviter, et plutôt le devinant que le voyant : c'est-à-dire [...] quelque chose d'insolite, d'irréel, d'hybride, en ce sens que ce qui avait été un cheval (c'est-à-dire ce qu'on savait, ce qu'on pouvait reconnaître, identifier comme ayant été un cheval) n'était plus à présent qu'un vague tas de membres, de corne, de cuir et de poils collés, aux trois quarts recouverts de boue -

[...] et quelque chose à l'intérieur de lui-même achevant de se désagréger, secoué par une sorte de terrifiante diarrhée qui le vidait sauvagement de son contenu comme de son sang même, et non pas morale, comme disait Blum, mais, pour ainsi dire mentale, c'est-à-dire non plus une interrogation, un doute, mais plus aucune matière à interrogation, à doute, disant tout haut (Georges) : "Mais le général aussi s'est tué [...] alors quand il a appris, c'est-à-dire s'est rendu compte, a fini par comprendre que sa brigade n'existait plus, avait été non pas anéantie, détruite selon les lois - ou du moins ce qu'il pensait être les lois - de la guerre: normalement, correctement, comme, par exemple [...], ou même encore - cela il l'eût peut-être, à la rigueur, admis - submergée par une attaque ennemie : mais pour ainsi dire absorbée, diluée, dissoute, bue, effacée de la carte d'état major sans qu'il sût où, ni comment, ni à quel moment [...].

Dans cette forme associant de façon "chimiquement pure" si l'on peut dire, le dire et l'écart - en ce que (on l'a vu) l'écart s'y dit dans une forme qui, à la lettre, ne dit que le dire - affleure, répétitivement, au plan second, du commentaire, la tension qui emporte le "courant" de paroles de ce texte : au glissement incessant, à la confusion, l'abolition des repères, des formes, des distinctions de lieux, de temps, de personnes, de matières et d'éléments - le vivant et le minéral, le solide et le liquide... -, menant répétitivement aux "gouffres" de "l'évanouissement de l'escadron" et de l'horreur de la "dissolution" de "ce qui avait été un cheval", opposer l'acharnement, la nécessité, - besoin et devoir - de dire, de nommer l'insaisissable, l'incompréhensible, l'impensable, et, éprouvant incessamment que ce réel échappe, se vouer non pas à le "fixer" mais seulement - vitalement - à "l'ainsi dire". 


\section{BIBLIOGRAPHIE}

AUTHIER-REVUZ, J. (1992) Les non-coïncidences du dire et leur représentation méta-énonciative - Etude linguistique et discursive de la modalisation autonymique, Thèse de Doctorat d'Etat, Université de Paris VIII - Saint-Denis.

AUTHIER-REVUZ, J. (1995) Ces mots qui ne vont pas de soi : Boucles réflexives et non-coïncidences du dire , Collection Prix Larousse, Ed. Larousse, Paris, 2 vol.

BARTHES, R. (1978) Leçon, Ed. du Seuil, Paris.

BORGES, J.L. (1983) Funes ou la mémoire (1944), in Fictions, trad. française Ed. Gallimard, Paris, coll. Folio.

HAAR, M. (1978) Nietsche et la maladie du langage, Revue philosophique, 4, oct-déc. 78, pp. 403-417.

LECLAIRE, S. (1971) Démasquer le réel - Un essai sur l'objet en psychanalyse, Editions du Seuil, Paris, Coll. Le champ freudien.

OSTER, D. (1983) Passages de Zénon, Editions du Seuil, Paris.

SCHNEIDER, M. (1985) Voleurs de mots, Editions Gallimard, Collection "Connaissance de l'inconscient", Paris.

\section{NOTES}

1. Une première version de ce texte est parue en néerlandais dans le cadre des manifestations "Antwerpen 93" sous le titre Gebrek van het zeggen, Zeggen van het gebrek: de woorden van de stilte in Vertoog en Literatuur, cahier 2, Woordenloosheid, Ed. Kritak (Leuven) et Meulenhoff (Amsterdam) 1993, p.55-74; elle a été reprise, en portugais, in Gestos de Leitura, Eni Orlandi (ed.), Ed. de l'UNICAMP, 1994.

Le terme de silence, où joue sans doute l'écho d'échanges amicaux avec Eni Orlandi, est pris ici seulement comme synonyme métaphorique du manque, inhérent à la non-coïncidence mot-chose, avec une valeur très restreinte par rapport à la théorisation dont la notion de silence comme fondatrice du sens fait l'objet dans le travail d'Eni Orlandi, récemment traduit en français : Les formes du silence, Ed. des Cendres, 1996.

2. Leclaire (71), p. 97.

3. Haar (78).

4. Borges, 1942, in (83), p. 117.

5. Barthes (78), p. 22.

6. Oster, (83), p. 190.

7. Schneider (85), p. 389.

8. E. Feydeau.

9. Configuration de la modalité autonymique, étudiée systématiquement dans Authier-Revuz (92) et (95).

10. La question du rapport mot-chose ne suscite pas l'unique réponse méta-énonciative évoquée ici - celle d'un dire explicitant le défaut du dire -, mais aussi celle de l'affirmation de la coïncidence réalisée (c'est le mot; je dis bien, ...) ou des figures variées et complexes de la 
nomination "entre deux mots" (X, j'ai failli dire $Y$; X, que dis-je $Y$; ...), cf. Authier-Revuz (95), chapitre 7.

\section{RÉSUMÉS}

Comment se dit, au fil du dire, le défaut du dire ? Sont parcourus ici les types de représentation de ce défaut d'abord (I) comme présence d'une imperfection placée au plan (a) du mode de dire (ex. : pour faire vite, ...) ou (b) du mot (ex. : le mot ne convient pas, ...), puis (II) comme absence (a) de l'énonciateur à son dire (ex. : j'ose à peine dire $X, \ldots),(b)$ du mot (ex. : il n'y a pas de mot, ...) et est interrogée la forme pour ainsi dire qui, ne disant que le fait de dire, est pourtant forme spécifique et stéréotypique du dire du défaut.

A travers la place - importante et différente - qu'elles font à ces formes, sont envisagées trois "écritures de l'écart" : P. Quignard, N. Sarraute, C. Simon.

What are the figures of speech speaking of the inadequacy of speaking? The inadequacy is represented (I) as the presence of an imperfection situated (a) in the way of speaking (ex. : to cut it short) or in the element itself (ex.: this is not the appropriate word), (II) as an absence, (a) the speaker missing somehow in his act of speaking (ex. : dare I say) or, (b) the right word is missing (ex. : the word does not exist), and (III) through the expression so to speak, which, expressing only the fact of speaking appears nevertheless as the prototypical form of the inadequacy of speech.

Three "writings in the gap" : P. Quignard, N. Sarraute, C. Simon, are described through the place important and different - they give to these representations of inadequacy.

\section{AUTEUR}

\section{JACQUELINE AUTHIER-REVUZ}

(Centre de Linguistique Française, Université de Paris III)

22, rue Montorgueil

F 78120 RAMBOUILLET 